\title{
On Galleries with No Bad Points*
}

\author{
P. Valtr \\ Department of Applied Mathematics, Charles University, \\ Malostranské nám. 25, 11800 Praha 1, Czech Republic \\ valtr@kam.ms.mff.cuni.cz \\ and \\ DIMACS Center, Rutgers University, \\ P.O. Box 1179, Piscataway, NJ 08855, USA \\ valtr@dimacs.rutgers.edu
}

\begin{abstract}
For any $k$ we construct a simply connected compact set (art gallery) in $\mathbb{R}^{3}$ whose every point sees a positive fraction (in fact, more than $\frac{5}{9}$ ) of the gallery, but the whole gallery cannot be guarded by $k$ guards. This disproves a conjecture of Kavraki et al.
\end{abstract}

\section{Introduction}

Consider an art gallery (i.e., compact set) $X$ of Lebesgue measure 1 in $\mathbb{R}^{d}, d \geq 2$, such that a guard placed anywhere in the gallery sees a region of Lebesgue measure at least $\varepsilon$. Kavraki et al. [3] conjectured that if $X$ has at most $h$ holes, then it can be guarded by at most $f_{d}(h, \varepsilon)$ guards, for some function $f_{d}$ polynomial in $h$ and $1 / \varepsilon$.

Kalai and Matoušek [2] proved a weaker form of the planar version of the conjecture (their function $f_{2}$ was not polynomial in $h$ ). Following some ideas of Kalai and Matoušek, the author [5] proved the planar version of the conjecture with $f_{2}(h, \varepsilon)=$ $(2+o(1))(1 / \varepsilon) \log (1 / \varepsilon) \log _{2}(h+2)$. Kalai and Matoušek [2] gave an example showing that $f_{2}\left(h, \varepsilon_{0}\right)=\Omega(\log h)$ for some fixed $\varepsilon_{0}>0$. Considering $\left\lfloor\varepsilon_{0} / \varepsilon\right\rfloor$ shifted copies of the set in their example, an example showing the lower bound $f_{2}(h, \varepsilon)=\Omega((1 / \varepsilon) \log h)$ for $\varepsilon \in\left(0, \varepsilon_{0}\right)$, which almost matches the above upper bound, can be obtained.

In this paper we disprove the conjecture of Kavraki et al. for $d \geq 3$. For any $k \geq 1$, we construct a simply connected gallery in $\mathbb{R}^{3}$, in which each point sees more than $\frac{\overline{5}}{9}$ of

\footnotetext{
* This research was supported by DIMACS Center, by Czech Republic Grant GAČR 201/1996/0194, and by Charles University Grants Nos. 193/1996 and 194/1996.
} 
the gallery, but the whole gallery cannot be guarded by $k$ guards. A motivation for the problem coming from robot path planning can be found in [3].

Let $X$ be a set in $\mathbb{R}^{d}, d \geq 2$. We say that a point $x \in X$ sees a point $y \in X$ in $X$ if the line segment $x y$ is fully contained in $X$. For $x \in X$, let $V(x)=\{y \in X: x$ sees $y\}$. We say that a subset $G \subseteq X$ guards $X$ if each point of $X$ sees at least one point of $G$. We say that a point $x \in X$ is $\varepsilon$-good, if $\lambda_{d}(V(x)) \geq \varepsilon \lambda_{d}(X)$, where $\varepsilon>0$ and $\lambda_{d}$ denotes Lebesgue measure in $\mathbb{R}^{d}$. We say that the set $X$ is $\varepsilon$-good, if each point of $X$ is $\varepsilon$-good.

Here is the main result:

Theorem 1. For any $k \geq 1$, there is a $\frac{5}{9}$-good simply connected compact set in $\mathbb{R}^{3}$ which cannot be guarded by $k$ points (guards).

In the proof of Theorem 1 we show that the constant $\frac{5}{9}$ can be slightly improved. It is easy to modify our construction so that it gives a $\frac{5}{9}$-good simply connected compact set in $\mathbb{R}^{3}$ which cannot be guarded by finitely many points (guards). An easy generalization of our construction also gives, for any $d \geq 3$, a simply connected compact $\left(1-((d-1) / d)^{d-1}\right)$-good gallery in $\mathbb{R}^{d}$ which cannot be guarded by a finite number of guards.

On the other hand, a nontrivial upper bound in any dimension follows from a wellknown theorem of Krasnosel'skij. Krasnosel'skij's theorem [4] says that if $X \subset \mathbb{R}^{d}$ is a connected compact set in $\mathbb{R}^{d}$ with the property that every $d+1$ points in $X$ can be seen from a single point in $X$, then the whole set $X$ can be seen from a single point, i.e., $X$ is star-shaped. Since the assumptions of Krasnosel'skij's theorem are obviously satisfied for any $(d /(d+1)+\omega)$-good compact set $X(\omega>0)$, the following upper bound follows:

Theorem 2. For $d \geq 2$ and $\omega>0$, any $(d /(d+1)+\omega)$-good compact gallery in $\mathbb{R}^{d}$ can be guarded by one guard (in other words, it is star-shaped).

There is a significant gap between the lower bound $1-((d-1) / d)^{d-1} \approx 1-1 / e$ in the $d$-dimensional version of Theorem 1 and the upper bound $d /(d+1)$ in Theorem 2 . In particular, we do not have an answer to the following question.

Problem 1. Is there an $\varepsilon<1$ such that for any $d \geq 2$ any (simply connected) $\varepsilon$-good compact gallery in $\mathbb{R}^{d}$ can be guarded by at most $k=k(d)$ guards?

We can partially solve Problem 1 if other Borel measures than the uniform (Lebesgue) one are considered. This is shown in Section 3. Section 2 is devoted to the proof of Theorem 1. Throughout the paper, $B(x, r)$ denotes the $r$-neighborhood of $x$.

\section{The Construction}

In this section we prove Theorem 1 . Let $S$ be a regular tetrahedron of edge length 1 with vertices $A, B, C, D$, let $U$ be the center of $S$, and let $V$ be the center of the triangle 


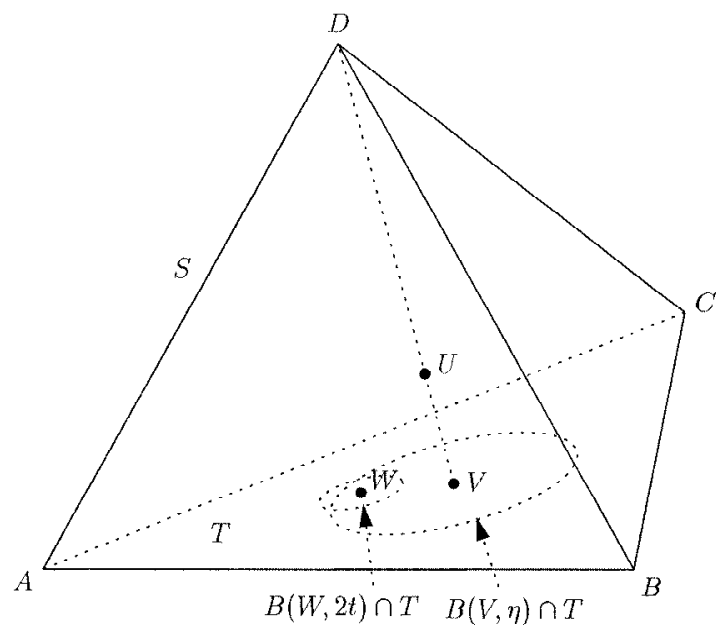

Fig. 1. The tetrahedron $S$.

$T=A B C$. We obtain the desired set from $S$ by removing small simplicial regions at the boundary of $S$. The following key lemma shows that, roughly speaking, we can remove a small region near a boundary point $W$ so that $W$ will not see relatively large neighborhoods of $k$ given points (see Fig. 1).

Lemma 1. For every $k \geq 1, \eta, \delta \in(0,0.01), W \in(B(V, \eta) \cap T), t>0, P_{1}, \ldots, P_{k} \in$ $S \backslash B(W, 2 t)$, there exists a set (union of $k$ tetrahedra) $S^{\prime \prime}=S^{\prime \prime}\left(\eta, \delta, W, t,\left\{P_{1}, \ldots, P_{k}\right\}\right)$ $\subset S$ such that

(i) $S^{\prime \prime} \subset B(W, 2 t) \backslash\{W\}$,

(ii) $S \backslash S^{\prime \prime}$ is simply connected and $\left(\frac{5}{9}-h(\eta, \delta)\right)$-good, where $h(\eta, \delta)$ is independent of $W, t,\left\{P_{1}, \ldots, P_{k}\right\}$ and $h(\eta, \delta) \rightarrow 0$ as $\eta+\delta \rightarrow 0$,

(iii) $W$ sees no point of $\bigcup_{i=1}^{k} B\left(P_{i}, \delta^{2} \cdot \operatorname{dist}\left(P_{i}, W\right)\right)$ in $S \backslash S^{\prime \prime}$.

Before proving Lemma 1 we complete the proof of Theorem 1.

Proof of Theorem 1. First we show a slightly weaker result. For any positive integer $k$ and for any real number $\omega>0$, we find a $\left(\frac{5}{9}-\omega\right)$-good simply connected gallery in $\mathbb{R}^{3}$ which cannot be guarded by $k$ guards.

Choose $\eta, \delta \in(0,0.01)$ such that $h(\eta, \delta)<\omega / 2$, where $h$ is the function from condition (ii) in Lemma 1. Fix a finite number of points $P_{1}, \ldots, P_{n}$ in $S$ such that $S \subset$ $\bigcup_{i=1}^{n} B\left(P_{i}, \delta^{2} \eta / 2 \sqrt{k}\right)$, and denote $I=\{1, \ldots, n\}$. For each $i \in I, T \cap B\left(P_{i}, \eta / 2 \sqrt{k}\right)$ is a disk of area at most $\pi \eta^{2} / 4 k$. Thus, the area of $\bigcup_{i \in J}\left(T \cap B\left(P_{i}, \eta / 2 \sqrt{k}\right)\right)$ is at most $\pi \eta^{2} / 4$ for each $J \in\left(\begin{array}{l}I \\ k\end{array}\right)$, where $\left(\begin{array}{l}I \\ k\end{array}\right)$ denotes the set of all $k$-element subsets of $I$. Since the area of $T \cap B(v, \eta)$ is $\pi \eta^{2}$, for any $J \in\left(\begin{array}{l}I \\ k\end{array}\right)$ we can fix a point $W(J)$ in $T \cap B(V, \eta)$ such that $\operatorname{dist}\left(P_{i}, W(J)\right)>\eta / 2 \sqrt{k}$ holds for every $i \in J$. Moreover, the points $W(J), J \in\left(\begin{array}{l}I \\ k\end{array}\right)$, can be taken pairwise distinct. 
For any $J \in\left(\begin{array}{l}I \\ k\end{array}\right)$ and $t \in(0, \eta / 4 \sqrt{k})$, there is a set $S^{\prime \prime}\left(\eta, \delta, W(J), t,\left\{P_{i}: i \in J\right\}\right)$ satisfying Lemma 1 (in particular, $S \backslash S^{\prime \prime}\left(\eta, \delta, W(J), t,\left\{P_{i}: i \in J\right\}\right)$ is $\left(\frac{5}{9}-\omega / 2\right)$-good since $h(\eta, \delta)<\omega / 2)$. It is easy to see that the compact set

$$
X=\overline{\bigcap_{J \in\left(\begin{array}{l}
I \\
k
\end{array}\right)} S \backslash S^{\prime \prime}\left(\eta, \delta, W(J), t,\left\{P_{i}: i \in J\right\}\right)}
$$

is simply connected and $\left(\frac{5}{9}-\omega\right)$-good for a sufficiently small $t>0$. We claim that $X$ cannot be guarded by $k$ guards. Suppose to a contrary that $X$ is guarded by a set $G$ of $k$ guards. Certainly, $G \subset \bigcup_{i \in J} B\left(P_{i}, \delta^{2} \eta / 2 \sqrt{k}\right)$ for some $J \in\left(\begin{array}{l}I \\ k\end{array}\right)$. Then, by condition (iii) in Lemma 1 , the point $W(J) \in X$ sees no point of

$$
\bigcup_{i \in J} B\left(P_{i}, \delta^{2} \operatorname{dist}\left(P_{i}, W(J)\right)\right) \supset \bigcup_{i \in J} B\left(P_{i}, \frac{\delta^{2} \eta}{2 \sqrt{k}}\right) \supset G
$$

in $X$, a contradiction.

To get a $\frac{5}{9}$-good set from the $\left(\frac{5}{9}-\omega\right)$-good set $X$, it suffices to remove a proper (quite small) region from $X$ in the neighborhood of the center of each of the triangles $A B D, A C D$, and $B C D$. For example, in the neighborhood of the center $V_{0}$ of the triangle $T_{0}=A B D$, we can remove the tetrahedron $\operatorname{conv}\left(T^{\prime} \cup\left\{U_{0}\right\}\right)$, where $T^{\prime} \subset T_{0}$ is the equilateral triangle with center $V_{0}$ and with sides of length $\frac{1}{10}$ parallel to those of $T_{0}$, and $U_{0}$ is the point on the segment $U V_{0}$ at distance $\frac{1}{100}$ from $V_{0}$. Similarly, we can remove the other two regions. In this way, if $\omega>0$ is sufficiently small, we even obtain a $\left(\frac{5}{9}+c\right)$-good simply connected gallery which cannot be guarded by $k$ guards $(c>0$ is a constant).

It remains to prove Lemma 1. Our proof of Lemma 1 relies on the following observation (see Fig. 2).

Observation 1. For every $\varepsilon<\frac{5}{9}$, there are two real numbers $\eta>0$ and $\alpha>0$ such that the following holds. Let $T^{\prime}$ be an equilateral triangle contained in $T \cap B(V, \eta)$,

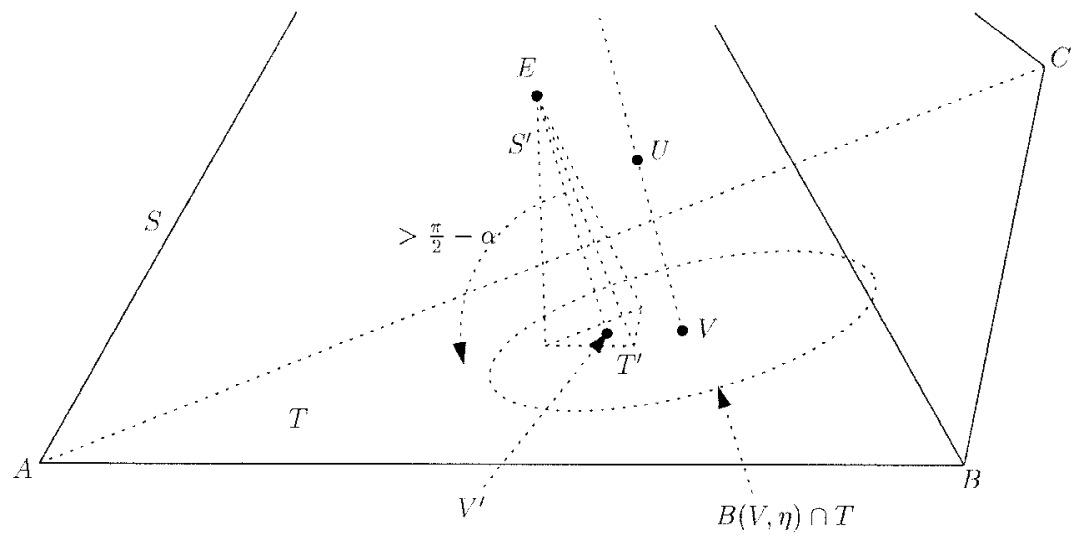

Fig. 2. The tetrahedron $S^{\prime}$. 


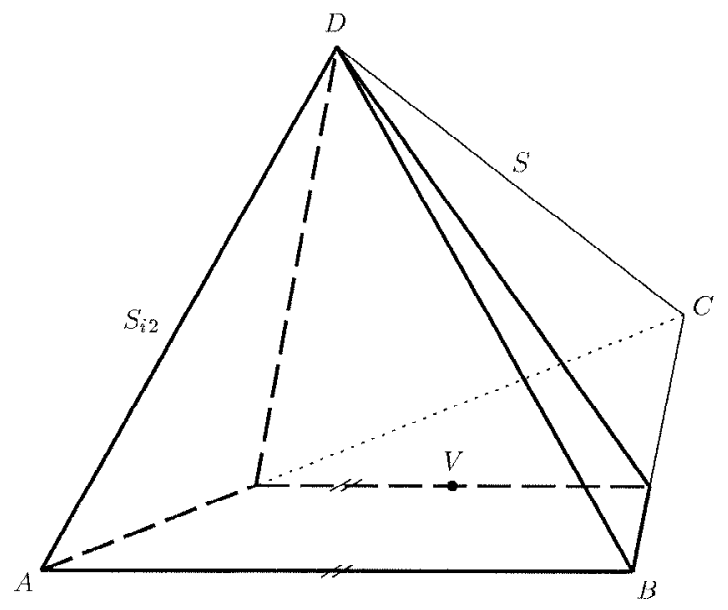

Fig. 3. One of the regions $S_{i 2} \subset S$ of measure $\frac{5}{9} \lambda_{3}(S)$.

with sides parallel to those of $T$. Let $V^{\prime}$ be the center of $T^{\prime}$, and let $E$ be a point inside $S$ such that the angle between the line $V^{\prime} E$ and the plane containing $T$ is bigger than $\pi / 2-\alpha$. Then the set $S \backslash S^{\prime}$, where $S^{\prime}=\operatorname{conv}\left(T^{\prime} \cup\{E\}\right)$, is $\varepsilon$-good.

Proof. Let $\varrho_{1}, \varrho_{2}, \varrho_{3}$ be the three planes containing the points $V, D$ and intersecting the triangle $T=A B C$ in a line segment parallel to a side of $T$. Then each $\varrho_{i}$ cuts the tetrahedron $S$ into two parts $S_{i 1}$ and $S_{i 2}$ of measure $\frac{4}{9} \lambda_{3}(S)$ and $\frac{5}{9} \lambda_{3}(S)$, respectively (see Fig. 3). If $\eta>0$ and $\alpha>0$ are small, then every point in $S \backslash S^{\prime}$ sees in $S \backslash S^{\prime}$ almost entirely one of the three regions $S_{i 2} \cap\left(S \backslash S^{\prime}\right)$ of measure $\approx \frac{5}{9} \lambda_{3}\left(S \backslash S^{\prime}\right)$. The observation follows.

Observation 1 yields the following weaker version of Lemma 1.

Lemma 2. For every $\eta, \delta \in(0,0.01), W \in(B(V, \eta) \cap T), t>0, P \in S \backslash B(W, 2 t)$, there exist a tetrahedron $S^{\prime}=S^{\prime}(\eta, \delta, W, t, P) \subset S$ and a real number $t^{\prime}=$ $t^{\prime}(\eta, \delta, W, t, P)>0$ such that

(i) $S^{\prime} \subset B(W, 2 t) \backslash B\left(W, 2 t^{\prime}\right)$,

(ii) $S \backslash S^{\prime}$ is simply connected and $\left(\frac{5}{9}-g_{1}(\eta, \delta)\right)$-good, where $g_{1}(\eta, \delta)$ is independent of $W, t, P$ and $g_{1}(\eta, \delta) \rightarrow 0$ as $\eta+\delta \rightarrow 0$,

(iii) every point of $(S \backslash B(W, 2 t)) \cup B\left(W, 2 t^{\prime}\right)$ is $\left(1-g_{2}(\delta)\right)$-good in $S \backslash S^{\prime}$, where $g_{2}(\delta)$ is independent of $W, t, P$ and $g_{2}(\delta) \rightarrow 0$ as $\delta \rightarrow 0$,

(iv) $W$ sees no point of $B\left(P, \delta^{2} \cdot \operatorname{dist}(P, W)\right)$ in $S \backslash S^{\prime}$.

Proof. Let $\eta, \delta, W, t, P$ satisfy the conditions of Lemma 2. Consider a plane $\rho$ containing the points $W, P$ and perpendicular to the plane $\tau$ containing $T$ ( $\rho$ is uniquely determined unless the line $W P$ is perpendicular to $\tau$ ). Choose the Cartesian coordinate system in $\rho$ so that the line $\rho \cap \tau$ coincides with the $x$-axis, $W$ coincides with the origin, and each of the two coordinates of $P$ is nonnegative. Denote $W^{\prime}=(\delta t, 0), E=(-\delta t, t)$, 


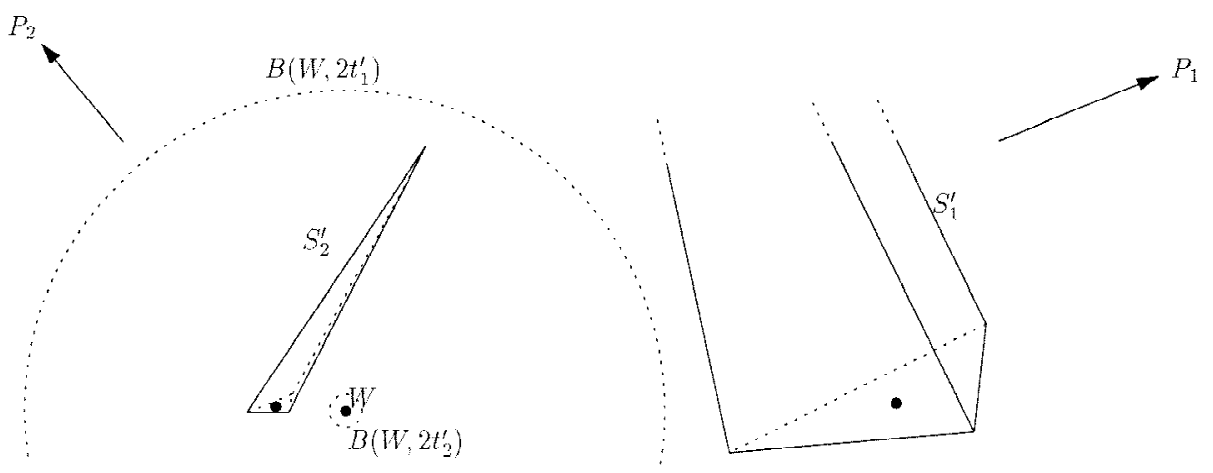

Fig. 4. The set $S_{2}^{\prime}$ consisting of two tetrahedra.

and return to $\mathbb{R}^{3}$. Let $T^{\prime} \subset T$ be the equilateral triangle with center $W^{\prime}$ and with sides of length $10 \delta^{2} t$ parallel to those of $T$. Then $S^{\prime}=\operatorname{conv}\left(T^{\prime} \cup\{E\}\right)$ satisfies the lemma: (i) and (iii) hold with $t^{\prime}=\delta^{2} t$, (ii) follows from Observation 1, and (iv) follows from the construction.

Repeated applications of Lemma 2 give the desired Lemma 1.

Proof of Lemma 1. Given $k, \eta, \delta, W, t,\left\{P_{1}, \ldots, P_{k}\right\}$, an application of Lemma 2 with parameters $\eta, \delta, W, t, P=P_{1}$ gives a set $S^{\prime}\left(\eta, \delta, W, t, P_{1}\right)=S_{1}^{\prime}$ and a number $t^{\prime}\left(\eta, \delta, W, t, P_{1}\right)=t_{1}^{\prime}>0$. Thereafter, an application of Lemma 2 with parameters $\eta, \delta, W, t=t_{1}^{\prime}, P=P_{2}$ gives a set $S^{\prime}\left(\eta, \delta, W, t_{1}^{\prime}, P_{2}\right)=S_{2}^{\prime}$ and a number $t^{\prime}\left(\eta, \delta, W, t_{1}^{\prime}, P_{2}\right)=t_{2}^{\prime}>0$ (see Fig. 4). We then apply Lemma 2 an additional $k-2$ times. In the $i$ th application $(i=3,4, \ldots, k)$, we use parameters $\eta, \delta, W, t=t_{i-1}^{\prime}, P=P_{i}$, and obtain a set $S^{\prime}=S_{i}^{\prime}$ and a number $t^{\prime}=t_{i}^{\prime}>0$. It is easily verified that the set $S^{\prime \prime}=\bigcup_{i=1}^{k} S_{i}^{\prime}$ satisfies Lemma 1 .

\section{Related Results}

In this section we prove some related results for other Borel measures than the uniform (Lebesgue) one. A Borel measure $\mu$ on $\mathbb{R}^{d}$ is called locally finite if $\mu(K)$ is finite for any compact measurable set $K \subset \mathbb{R}^{d}$. Let $X$ be a set in $\mathbb{R}^{d}, d \geq 2$, and let $\mu$ be a Borel measure on $\mathbb{R}^{d}$. We say that a point $x \in X$ is $(\mu, \varepsilon)$-good, if $\mu(V(x)) \geq \varepsilon \mu(X)$. We say that the set $X$ is $(\mu, \varepsilon)$-good, if each point of $X$ is $(\mu, \varepsilon)$-good. We call a set in $\mathbb{R}^{d}$ polyhedral if it is a union of finitely many convex $d$-dimensional polytopes. An analogue of Theorem 2 for locally finite Borel measures gives almost a tight result.

Theorem 3. Let $\omega>0$. Then

(i) for any locally finite Borel measure $\mu$ in $\mathbb{R}^{d}, d \geq 2$, any polyhedral $(\mu, d /(d+1)+\omega)$-good set of positive $\mu$-measure in $\mathbb{R}^{d}$ can be guarded by one guard, 
(ii) for any $d \geq 3$, there is a locally finite Borel measure $\mu_{d}$ in $\mathbb{R}^{d}$ such that for any $k \geq 1$ there is a polyhedral $\left(\mu_{d},(d-1) / d-\omega\right)$-good simply connected gallery of positive $\mu_{d}$-measure which cannot be guarded by $k$ guards.

Thus, an analogue of the question in Problem 1 for locally finite Borel measures has a negative answer. We remark that in the plane the number of guards needed in a $(\mu, \varepsilon)$-good gallery with $h$ holes is bounded by $(2+o(1))(1 / \varepsilon) \log (1 / \varepsilon) \log _{2}(h+2)$ for any locally finite Borel measure $\mu$, like for the uniform measure. This is because the proof in [5] for the uniform (Lebesgue) measure does not use any specific properties of the uniform measure, and therefore can be applied for any locally finite Borel measure.

Proof of Theorem 3. (i) Let $d \geq 2, \omega>0$, and let $\mu$ be a locally finite Borel measure in $\mathbb{R}^{d}$. Let $X$ be a $(\mu, d /(d+1)+\omega)$-good polyhedral gallery in $\mathbb{R}^{d}$ with $\mu(X)>0$. Let $P$ be a centerpoint ${ }^{1}$ of $X$ with respect to $\mu$. We claim that $P$ sees every point in $X$. Suppose to contrary that $P$ does not see a point $Q \in X$. Let $Q^{\prime} \in X$ be the point of $X$ on the segment $P Q$ closest to $P$ with the property that it does not see $P$. Then there is a point $Q^{\prime \prime}$ of $X$ in a small neighborhood of $Q^{\prime}$ which sees a part of $X$ contained in a closed halfspace not containing $P$. From the centerpoint property of $P$, this implies that $Q^{\prime \prime}$ is not $(\mu, d /(d+1)+\omega)$-good, a contradiction.

(ii) We outline the construction for $d=3$. The construction is analogous for the other values of $d$. For $d=3$, the gallery is similar to the gallery from the proof of Theorem 1 . We again consider a regular tetrahedron $S=A B C D$ with edge length 1 . Consider the Borel measure $\mu_{3}$ which is the Lebesgue measure on the $\frac{1}{10}$-neighborhood of each of the vertices $A, B, C$, and the zero measure on the rest of $\mathbb{R}^{3}$. The proof now goes literally as the proof of Theorem 1 in Section 2 (including Observation 1 and Lemmas 2 and 1) with the last paragraph omitted and with the exceptions that every occurrence of $\frac{5}{9}$ is replaced by $\frac{2}{3}$, and that the measure $\mu_{3}$ is considered instead of the Lebesgue measure (thus, the notion $\varepsilon$-good is always replaced by $\left(\mu_{3}, \varepsilon\right)$-good).

\section{Acknowledgments}

I would like to thank Jiří Matoušek for bringing this problem to my attention and for valuable discussions. I also thank an anonymous referee for many helpful comments and for pointing out Krasnosel'skij's theorem to me.

\section{References}

1. H. Edelsbrunner. Algorithms in Combinatorial Geometry. Springer-Verlag, Berlin (1987).

2. G. Kalai and J. Matoušek. Guarding galleries where every point sees a large area, Israel J. Math., 101 (1997), 125-139.

\footnotetext{
${ }^{1} P$ is a centerpoint of a compact set $X \subset \mathbb{R}^{d}$ with respect to a locally finite Borel measure $\mu$ if every halfspace in $\mathbb{R}^{d}$ containing $P$ contains a subset of $X$ of measure $\geq(1 /(d+1)) \mu(X)$. The existence of a centerpoint for any compact set $X \subset \mathbb{R}^{d}$ with respect to a locally finite Borel measure follows easily from the existence of a centerpoint for any finite set in $\mathbb{R}^{d}$ with respect to Lebesgue measure (see [1]).
} 
3. L. Kavraki, J.-C. Latombe, R. Motwani, and P. Raghavan. Randomized query processing in robot motion planning. Proc. 27th ACM Symp. Theoret. Comput. (1995), pp. 353-362.

4. M. A. Krasnosel'skij. Sur un critère pour qu'un domaine soit étoilé (in Russian, with French summary). Mat. Sb. (N.S.), 19(61) (1946), 309-310.

5. P. Valtr. Guarding galleries where no point sees a small area, Israel J. Math., 104 (1998), 1-16.

Received May 2, 1997, and in revised form February 5, 1998. 